\title{
USOS DA VIGILÂNCIA E DA MONITORIZAÇÃO EM SAÚDE PÚBLICA
}

\author{
Elisen Alves Waldman ${ }^{1}$
}

\begin{abstract}
Resumo
O objetivo deste texto é apresentar a vigilância e a monitorização como distintos instrumentos de saúde pública. A vigilancia apresenta, pelo seu elevado grau de desenvolvimento, uma perfeita delimitação de objetivos, métodos, fontes de dados e procedimentos de avaliaf̧ão. Tais particularidades the conferem, segundo alguns autores, a autonomia de uma disciplina em sauide pública. A vigilancia, porém, éum instrumento de menor abrangência do que a epidemiologia, pois, enquanto a primeira volta-se exclusivamente para 0 acompanhamento de especificos eventos adversos à saúde, a epidemiologia entendida como método ou como uma prática de saúde puiblica preocupa-se com o processo saúde-doença em populaçoes e seus determinantes. Entre as diferenças mais relevantes entre vigilancia e monitorização, temos que a vigilancia analisa exclusivamente o comportamento de eventos adversos a savide na cominidade, constituindo uma das aplicaçoes da epidemiologia em saúde puiblica, enquanto a monitorização acompanha indicadores e pode ser utilizada em diferentes áreas de atividade como, por exemplo, o acompanhamento de indicadores económicos, demográficos, de qualidade ambiental, etc. Entre as semelhanças apresentadas por esses dois instrumentos há a existéncia obrigatória de trếs componentes: a informação, a análise e a ampla disseminação da informação analisada a todos que dela necessitam.
\end{abstract}

Palavras Chave: Vigilancia em Saúde Pública; Monitorização; Epidemiologia em Serviços de Saúde.

\section{Summary}

The objective of the present text is to present surveillance and monitoring as two different public health tools. Surveillance is characterised by a high degree of development, leading to a precise delimitation of objectives, methods, sources of data, and evaluation procedures. According to some authors, these specific features give surveillance the autonomy of a Public Health Discipline. Surveillance is one of the applications of epidemiology in public health, and is concerned exclusively with the follow-up of specific health adverse events in a community, while epidemiology, either as a method or as a public health practice, is concerned with the health-disease process in populations and its determining factors. The most relevant differences between surveillance and monitoring are that surveillance is one of the applications of epidemiology in public health and analyses exclusively the behaviour of health adverse events in a community, while monitoring follows only indicators, it isn't an exclusive application of epidemiology, and may be used in different activities such as monitoring economic, demographic, environmental quality indicators, among others. Both, surveillance and monitoring have three obligatory components: information, analysis, and the widespread dissemination of the information analysed to all that need it.

Key Words: Public Health Surveillance; Monitoring; Epidemiology in Health Care Services.

\footnotetext{
${ }^{1}$ Departamento de Epidemiologia da Faculdade de Saúde Pública da Universidade de São Paulo. Endereço para correspondência: Av. Dr. Arnaldo, 715; São Paulo - SP / CEP: 01246-904 - Fax: (011) 881-2108 E-mail: eawaldman@usp.br
} 


\section{Introdução}

O uso da vigilância, especialmente nas últimas quatro décadas, foi significativamente ampliado, especialmente a partir do inicio da Campanha de Erradicação da Variola nos anos 60, quando sua utilização disseminou-se por todos os continentes, propiciando sua consolidação como um importante instrumento da epidemiologia nos serviços de saúde. ${ }^{l}$

Em virtude de essa experiência ter se desenvolvido em numerosos paises com distintos graus de desenvolvimento econômico $e$ dotados de diferentes tipos de estrutura $e$ organização dos sistemas de saúde, assistimos à incorporação da vigilância, nas diversas regióes do mundo, com características particulares, ora restrita ao papel de um sistema de informação para a agilização das ações de controle, confundindo-se com alguma freqüência com os próprios programas de controle de doenças transmissiveis, ora abrangendo a pesquisa e confundindo-se com a própria epidemiologia.

Apesar de essa diversidade de experiências, a vigilância no ocorrer desse período, atingin apreciável grau de delimitação em seus objetivos, fontes de dados, metodologia e procedimentos de avaliação. O nivel de especificidade atingido permitiu-lhe caracterizar-se como um instrumento de saúde pública de importante aplicação, seja na agilização das ações de controle de eventos adversos à saúde, seja no apoio ao esforço de permanente aperfeiçoamento técnico dos serviços de saúde para o estabelecimento de estratégias eficientes de controle de doenças.

Sua metodologia pode ser resumida pela atividade de acompanhamento continuo $e$ análise regular do comportamento de especificos eventos adversos à saúde em populações e pela elaboração, com fundamento no conbecimento científico, das bases técnicas que oferecem sustentação às estratégias adotadas pelos programas de controle desses eventos.

Apesar de perfeitamente delimitada como instrumento de saúde pública, tem sido freqüente a utilização do termo monitorização como sinônimo de vigilancia, o que nos parece equivocado, uma vez que ambos apresentam aplicações distintas em saúde pública. A monitorização, diferentemente da vigilância, não dispõe ainda de uma perfeita delimitação de seus objetivos, metodologia e critérios de avaliação de seu desempenho.

Se por um lado, a monitorização ainda não alcançou seu pleno desenvolvimento, verificamos, por outro, que sua aplicação é bem mais ampla do que a vigilância, uma vez que não se restringe à área da saúde, podendo ser utilizada em diferentes campos de atividade, entre eles o acompanbamento sistemático de indicadores econômicos, sociais, demográficos, de condições climáticas, de poluição ambiental, para citarmos alguns exemplos.

$A$ incorporação da monitorização no campo da saúde é recente. Provavelmente, os profissionais de unidades de terapia intensiva tenham sido os primeiros a utilizar esse instrumento ao coletarem, de forma sistemática, indicadores das condições vitais, de pacientes graves, com a finalidade de identificar sinais de alerta para a necessidade de intervenção. ${ }^{2}$

Em 1976, o "International Journal of Epidemiology"3 dedica um numero especial sobre a vigilancia, fato que reflete $o$ reconbecimento da importância e abrangência de sua aplicação em saúde pública. Em seu editorial são feitas várias considerações, entre elas a de que a vigilância requer a combinação e coordenação de uma boa coleta de dados por um pessoal motivado, um eficiente sistema de saúde, análise bem feita das informações com retorno rápido com objetivo de orientar as ações de controle. Comenta também a grande variedade de aplicações da vigilância, salientando, por outro lado, que a monitorização é um instrumento relativamente novo e que deve ser melhor estabelecida sua delimitação com a vigilancia.

A melhor delimitação da aplicação desses dois instrumentos no campo da saude, longe de constituir um tema de interesse eminentemente acadêmico, tem inegável valor prático, 
especialmente neste momento, quando vivemos uma profunda reestruturação de nosso sistema de saude, sendo indispensável a identificação dos instrumentos de saude pública que utilizaremos nesse processo e das caracteristicas e perfis dos recursos humanos a eles adequados, assim como a infra-estrutura e equipamentos indispensáveis, tendo em vista a necessidade de oferecermos um minimo de auto-sustentação ao Sistema Único de Saúde (SUS).

Para tornar mais clara a terminologia utilizada neste texto, estamos entendendo como instrumento de saude pública os recursos utilizados para atingir o objetivo de oferecer assistência integral à saúde à população. Citariamos, entre os instrumento de saude pública: a ação programática, o planejamento, a educação sanitária, a fiscalização sanitária, a vigilância, a monitorização, a pesquisa em saúde pública e a capacitação de recursos humanos. ${ }^{4}$

Neste momento em que visualizamos condições objetivas para um avanço qualitativo da aplicação da vigilância e, de forma mais ampla, da epidemiologia no ámbito do SUS, parece oportuna a discussão a respeito da melhor delimitação do uso da vigilancia $e$ da monitorização como instrumentos de saúde pública. Este é o objetivo da apresentação deste texto.

\section{Vigilância}

A vigilancia tem dois significados internacionalmente consagrados em saúde publica: um que poderíamos denominar de clássico, aplicado inicialmente nos fins do século passado, tem sua utilização vinculada aos conceitos de isolamento e quarentena. Conceitos que surgem no final da Idade Média e consolidam-se nos séculos XVII e XVIII com o fortalecimento do comércio e a proliferação de centros urbanos. ${ }^{5}$

$O$ isolamento $e$ a quarentena determinavam a separação de individuos de seus contatos habituais, assumindo caráter compulsório, típico da policia médica, visando defender as pessoas sadias, separando-as dos doentes ou daquelas que potencialmente poderiam vir a apresentar essa condição. Este conjunto de medidas de tipo restritivo, policial e com caráter punitivo, criava sérias dificuldades para o intercámbio comercial entre paises. ${ }^{5}$

O grande desenvolvimento do comércio internacional na segunda metade do século passado e, por outro lado, o desenvolvimento da microbiologia e o melhor conbecimento dos mecanismo de transmissão das doenças infecciosas levou à elaboração de um novo instrumento para o controle dessas doenças. Surge, então, em saúde pública o conceito de vigilancia, definido pela específica mas limitada função de observar contatos de pacientes atingidos por moléstias graves como a cólera, a variola e a peste. Seu propósito era detectar os primeiros sintomas para a rápida instituição do isolamento. ${ }^{6}$

Neste contexto vigilancia deve ser entendida como a "observação dos comunicantes durante o periodo máximo de incubação da doença, a partir da data do último contato com um caso clinico ou portador, ou da data em que o comunicante abandonou o local em que se encontrava a fonte primária da infeçã $o^{\prime \prime}$.

Em sintese, este conceito envolvia a manutenção do alerta responsável e a observação para que fossem tomadas as medidas indicadas no momento oportuno. Portanto, constituia uma conduta mais sofisticada e "democrática" do que a prática restritiva de quarentena. ${ }^{6}$

A partir da década de 1950 observamos a introdução de um novo conceito de vigiláncia aplicado à saúde pública, desta vez no sentido de acompanhamento sistemático de eventos adversos à saúde na comunidade, com o propósito de aprimorar as medidas de controle. A metodologia aplicada pela vigiláncia, nesse novo conceito, inclui a coleta sistemática de dados relevantes relativos a especificos eventos adversos à savide e sua contínua avaliação e disseminação a todos que necessitam conhecê-los. ${ }^{8}$

Alexander Langmuir, em 1963, definiu vigilância como a "observação contínua da distribuição e tendéncias da incidência de doenças 
mediante a coleta sistemática, consolidação $e$ avaliação de informes de morbidade e mortalidade, assim como de outros dados relevantes e a regular disseminação dessas informaçôes a todos que necessitam conhecê-la”.

Afirmava também o autor que esse novo conceito de vigilancia, assim como a sua forma de operacionalização, fundamenta-se na prática desenvolvida na Inglaterra, no século XIX, por William Farr. 9

A designação inicialmente adotada para esse instrumento foi a de inteligência epidemiológica. O termo inteligência pode aqui ser compreendido em seu significado mais amplo como "faculdade ou habilidade de aprender, apreender ou compreender" ou em seu sentido mais restrito, predominantemente militar, "obter e dispor de informações, particularmente das informações secretas". ${ }^{10}$

Talvez para evitar o estigma do caráter militar do termo inteligência, temos a sua substituição por vigilancia que é aplicado pela primeira vez em saúde pública, nessa nova concep̧̧ão, em 1955, quando algumas regiöes dos Estados Unidos da América (EUA) foram atingidas por uma epidemia de poliomielite, evento que ficou conbecido como o "Acidente de Cutter". Essa epidemia apresentou a particularidade de atingir crianças, assim como seus contatos, logo após as primeiras terem recebido a administração de vacina de vírus inativado (tipo salk) contra a poliomielite. ${ }^{11}$ Entre os resultados mais relevantes da vigilância da poliomielite, nesta oportunidade, tivemos a produção de novos conbecimentos a respeito de diferentes aspectos desta doença considerados até nossos dias, como básicos para seu controle. $A$ vigilancia adquirirá o qualificativo epidemiológica em 1964, num artigo sobre o tema publicado por Karel Raska, designação que será internacionalmente consagrada com a criação, no ano seguinte, da Unidade de Vigiláncia Epidemiológica da Divisão de Doenças Transmissiveis da Organização Mundial da Sande. ${ }^{12}$

Foege e colaboradores ${ }^{13}$ salientaram que a vigilancia tornou-se um instrumento universalmente utilizado, porém existe a necessidade contínua de seu aprimoramento à medida que a demanda por respostas torna-se cada vez mais complexa. Afirmam ainda que os sistemas de vigilancia caracterizam-se por ter, obrigatoriamente, três componentes: a coleta da informação, a análise de dados e a disseminação das informaçôes adequadamente analisadas.

Abordando aspectos conceituais, Langmuir $^{8}$ assinalou alguns pontos ao definir vigilância. Num deles, alertava para que não se confundisse vigilancia com as açoes de controle de doenças pois ambas constituem instrumentos distintos de saúde pública, salientando, ainda, que as ações de controle são de responsabilidade das autoridades locais de saude. Afirmava também que a vigilancia não devia ser entendida como sinônimo de epidemiologia, pois esta, seja como ciência ou como uma prática de saúde pública, é muito mais ampla do que a vigilância. Por fim, assinalava a importância da interface entre vigilancia, pesquisa epidemiológica e açôes de controle de doenças.

Langmuir $^{8}$ era favorável ao conceito de vigilancia entendida como uma das aplicações da epidemiologia em saúde pública a que denominava de inteligéncia epidemiológica. Dizia ele que o profissional que trabalba na vigilância deve assumir o papel dos "olhos e ouvidos da autoridade sanitária", assessorando-a quanto à necessidade de medidas de controle, porém a decisão e a operacionalização dessas medidas devem ficar sob a responsabilidade dessa autoridade.

Essas opiniões devem ser entendidas no contexto de um país onde é forte o respeito à autonomia das Unidades da Federação e onde, por decorrência, o poder de intervenção do governo federal sempre foi restrito. Logo, o fato da vigiláncia ter sido desenvolvida por um órgão federal, o Centro de Controle e Prevenção de Doenças (CDC), a sua atuação deveria caracterizar-se como a de uma agência técnica de referência. Nesta posição, o CDC não determina, mas, recomenda aos órgãos estaduais de sauide as estratégias mais adequadas para 0 controle de doenças, fundamentadas $\mathrm{em}$ 
conbecimentos cientificos rigorosamente atualizados. Os Estados, por sua vez, acatando ou não tais recomendaçôes, assumem perante a comunidade a responsabilidade pelos resultados obtidos.

Ainda a respeito de aspectos conceituais da vigilancia, merece destaque o extenso trabalho publicado em 1988 por Thacker e Berkelman. ${ }^{14}$ Nele os autores discutem, entre outros pontos, quais seriam os limites da prática da vigiláncia $e$ analisam se o termo epidemiológica era apropriado para qualificar vigilancia na forma em que ela é aplicada em saúde pública.

Afirmam esses autores que as informações obtidas como resultado da vigilancia podem ser usadas para identificar questões a serem pesquisadas, como é o caso de testar uma hipótese elaborada a partir de dados obtidos numa investigação de um surto, relativa a uma possivel associação entre uma exposição (fator de risco) e um efeito (doença); ou avaliar a necessidade de definir determinada estratégia de controle de uma doença. Porém, enfatizam, concordando com Langmuir, que a vigilancia não abrange a pesquisa epidemiológica nem as açôes de controle; estas trés práticas de saúde puiblica são relacionadas mas independentes. As atividades desenvolvidas pela vigilancia situam-se num momento anterior à implementacãa de pesquisas e à elaboração de programas voltados ao controle de eventos adversos à sanide. ${ }^{14}$

Nesse contexto, afirmam Thacker $e$ Berkelman, ${ }^{14}$ o uso do termo epidemiológica para qualificar vigilância é equivocado, uma vez que epidemiologia é uma disciplina abrangente, que incorpora a pesquisa e cuja aplicação nos serviços de saúde vai além do instrumento de savide puiblica que denominamos vigilancia. $A$ utilização desse qualificativo tem induzido freqüentemente a confusões que restringem a aplicação da epidemiologia nos serviços ao acompanhamento de eventos adversos à savide, atividade que constitui somente parte das aplicacooes da epidemiologia em saúde pública.

Em função dessa discussão, Thacker $e$ Berkelman ${ }^{14}$ propuseram a adoção da denominação de vigilância em saúde pública como forma de evitar confusões a respeito da precisa delimitação dessa prática.

Desde 1989, o termo vigilância epidemiológica foi substituido pela denominação vigilância em saúde pública, que consagrou-se internacionalmente, sendo utilizada em todas as publicações sobre o assunto, desde o inicio dos anos 90, inclusive em documento recente da Organização PanAmericana de Saúde. ${ }^{15}$ Cumpre salientar que essa alteração da denominação não implicou a adoção de uma nova abordagem ou modificaçóes de seus aspectos conceituais on operacionais da vigiláncia.

\section{Aspectos operacionais da vigilância}

Para não nos limitarmos a aspectos conceituais na caracterização da vigilância como instrumento de saúde pública, passaremos a apresentar de forma sucinta alguns aspectos operacionais que caracterizam a vigilância como uma das aplicações da epidemiologia nos serviços de saúde.

\section{Objetivos da vigilância}

Entre os principais objetivos da vigilância, podemos citar: ${ }^{16}$

- Identificar tendências, grupos e fatores de risco com vistas a elaborar estratégias de controle de especificos eventos adversos à saúde.

- Descrever o padrão de ocorrência de doenças de relevancia em saúde pública.

- Detectar epidemias.

- Documentar a disseminação de doenças.

- Estimar a magnitude da morbidade e mortalidade causadas por determinados agravos.

- Recomendar, com bases objetivas e científicas, as medidas necessárias para prevenir ou controlar a ocorrência de especificos agravos à saúde.

- Avaliar o impacto de medidas de intervenção.

- Avaliar a adequação de táticas e estratégias de aplicação de medidas de intervenção, não 
só nos seus fundamentos técnicos mas também naqueles referentes à própria operacionalização dessas intervenções.

Não podemos entender, como objetivo da vigilancia, meramente a coleta e análise das informaçôes mas também a responsabilidade de elaborar, com fundamento em conhecimentos cientificos rigorosamente atualizados, as bases técnicas que oferecerão subsidios aos serviços de saúde na elaboração e implementação dos programas de saúde com a preocupação de continuo aprimoramento, assim como a agilização da identificação de problemas de maneira a propiciar a oportuna intervenção para seu controle.

Fontes de dados utilizados pela vigilância

Mesmo sem entrar em pormenores a respeito das vantagens e desvantagens de cada uma das fontes mais utilizadas por sistemas de vigilancia, consideramos oportuno apresentar as mais freqüentemente utilizadas. Entre elas temos: ${ }^{16}$

I- Sistemas compulsórios de notificações de doenças.

II- Sistemas articulados de laboratório.

III- Dados hospitalares.

$I V$ - Eventos sentinelas.

$V$ - Médicos sentinelas.

VI- Unidades de assistência primária à saúde.

Tipos de sistemas de vigilância

Dependendo das características do agravo, dos objetivos do sistema, dos recursos disponiveis, da fonte ou das fontes de informação a serem utilizadas, podemos optar por sistemas ativos ou passivos de vigilancia. Para tomarmos a decisão devemos analisar as vantagens, desvantagens e limitações de cada uma dessas duas opções. ${ }^{16}$

\section{Sistemas passinos}

Os sistemas de vigilancia passivos caracterizam-se por ter como fonte de informação a notificação espontânea, constituindo a forma mais antiga $e$ freqüentemente utilizada na análise sistemática de eventos adversos à saúde e, além disso, apresentam menor custo e maior simplicidade. Porém, este tipo de vigilância tem a desvantagem de ser menos sensivel, ou seja, é mais vulnerável à subnotificação, portanto, menos representativo, apresentando maior dificuldade na padronização da definição de caso.

2. Sistemas ativos

Os sistemas ativos de vigilancia caracterizam-se pelo estabelecimento de um contato direto, a intervalos regulares, entre a equipe da vigilância e as fontes de informação, geralmente constituidas por clinicas públicas $e$ privadas, laboratórios e hospitais.

Essa forma de obtenção de dados é, geralmente, aplicada a doenças que ocorrem raramente ou em sistemas de vigilancia voltados aos programas de erradicação de doenças. Os sistemas ativos de coleta de informação permitem um melhor conbecimento do comportamento dos agravos à saúde na comunidade, tanto $\mathrm{em}$ seus aspectos quantitativos quanto qualitativos. No entanto, são geralmente mais dispendiosos, necessitando também uma melhor infraestrutura dos serviços de saude.

Na prática é freqüente a utilização de sistemas mistos, ou seja, sistemas passivos "parcialmente ativados". Poderiamos exemplificálos como sistemas passivos, porém "parcialmente ativados" pelo contato direto e regular com uma fonte ou um número reduzido de fontes de informação que centralizam o atendimento de grande número de casos do agravo objeto do sistema.

\section{Características gerais dos sistemas de vigilância}

Em função das peculiaridades de cada Sistema Nacional de Saúde (SNS), temos a aplicação da vigilancia com diferentes graus de abrangência, mas existem algumas caracteristicas desse instrumento que são internacionalmente aceitas, entre elas: ${ }^{4}$

1. Cada sistema de vigilância será responsável pelo acompanhamento contínuo de especificos eventos adversos à saúde, com o objetivo de estabelecer as bases técnicas para a elaboração $e$ 
implementação dos respectivos programas de controle. Cada sistema de vigilância, em função de seus objetivos e peculiaridades, apresentará caracteristicas particulares.

2.Os sistemas de vigilância devem ser simples e contimuos, apresentando, obrigatoriamente, três componentes: a) coleta de dados; b) análise; c) a ampla distribuição das informações analisadas a todos que as geraram e que delas necessitam tomar conbecimento. $O$ instrumento de divulgação das informações analisadas será o Boletim Epidemiológico.

3.A vigilância deve ser entendida como um prérequisito para elaboração de programas de saúde e um instrumento para avaliação do impacto de sua implementação. Deve ser útil também para identificação dos fatores de risco e das populações vulneráveis à exposição ao risco, de forma a tornar mais efetivas as medidas de controle.

4.Deve ser avaliado freqüentemente e, se necessário, alterado de maneira a garantir seu bom desempenho, adequando-o periodicamente às modificações da estrutura, grau de desenvolvimento e complexidade tecnológica do Sistema Nacional de Sauide.

\section{Indicadores de avaliação de desempenho} da vigilância

Apresentaremos a seguir, de forma sintética, os indicadores de avaliação de desempenho de sistemas de vigilância mais freqüentemente utilizados. ${ }^{14}$

- Utilidade: Esse atributo expressa, em resumo, se o sistema está alcançando seus objetivos.

- Oportunidade: É avaliada pela análise da agilidade do sistema em cumprir todas as suas etapas, desde a notificação do caso até a indicação de medidas de controle e, por fim, a distribuição das informações devidamente analisadas por meio dos Boletins Epidemiológicos.

- Aceitabilidade: É avaliada pela disposição favorável dos profissionais e das instituições que conduzem o sistema, permitindo que as informações geradas sejam exatas, consistentes e regulares.
- Simplicidade: Os sistemas de vigilância, quando simples, são fáceis de compreender $e$ de implementar e pouco dispendiosos. Uma representação gráfica do sistema, apresentando o fluxo de informaçôes $e$ de respostas, poderá facilitar a avaliação desse atributo.

- Flexibilidade: Pode ser analisada pela habilidade de um sistema de vigiláncia adaptar-se facilmente a novas necessidades, em resposta às mudanças na natureza ou na importância de um evento adverso à saúde.

- Representatividade: A notificação dos casos obtidos por um sistema de vigiláncia é raramente completo. Os casos notificados podem diferir dos não notificados em suas caracteristicas demográficas, local ou uso de serviços de saúde ou exposição a riscos. Um sistema de vigilancia representativo descreve, com exatidão, a ocorrência de um evento adverso à saúde, ao longo do tempo, segundo os atributos da população e a distribuição espacial dos casos.

- Sensibilidade: Esse atributo pode ser avaliado pela capacidade de um sistema de vigiláncia identificar os verdadeiros casos do evento adverso à saúde que tem por objetivo acompanbar e analisar.

- Valor preditivo positivo (VPP): Esse atributo pode ser entendido como a proporção de individuos identificados como casos pelo sistema de vigilancia, e que de fato o são. Um sistema de vigilância de baixo VPP, ou seja, que apresente freqüentes confirmações de casos falsamente positivos, além de elevar seus custos, pode induzir à investigação de epidemias que de fato não ocorreram.

Resumindo, cabe salientar que os diversos atributos apresentados são interdependentes $e$ o aprimoramento de um pode comprometer outro. Quando aumentamos a sensibilidade de um sistema para detectar uma grande proporção de casos de um determinado evento adverso à saúde, estaremos, geralmente, também aprimorando a representatividade e a utilidade do sistema. Porém, nesse caso, teremos um aumento do custo, e aumento dos casos falsopositivos e, geralmente, uma diminuição da especificidade. 
Portanto, antes de recomendar modificações em um sistema de vigilância, deveremos observar as interações dos seus diversos atributos e custos, de forma a garantir que o aprimoramento de uma característica não afete negativamente a outra, a ponto de comprometer o desempenho global do sistema. Por fim, é importante enfatizar que o objetivo primordial do aprimoramento de sistema de vigilância é tornar mais efetivas e ágeis as medidas de controle de eventos adversos à sauide.

Vale assinalar que o desenvolvimento de indicadores de avaliação de desempenho de sistemas de vigilancia constituiram o principal passo para a consolidação da vigilância na qualidade de uma das principais aplicaçôes da epidemiologia nos serviços de saúde; alguns autores chegam a classificar a vigilância como uma disciplina completa no campo da saúde pública. ${ }^{17}$

\section{A vigilância em um sistema descentralizado de saúde.}

Utilizando o enfoque sistêmico $e$ sintetizando as diversas experiências $e$ concepçôes de vigiláncia desenvolvidas nos uiltimos 40 anos em diferentes partes do mundo, sem discutir o mérito de cada uma delas em particular, mas com a preocupação de estabelecer uma proposta adequada, em suas linhas gerais, a um sistema de saúde descentralizado e com um desempenho que lhe confira um minimo de auto-sustentação técnica, podemos dizer que a vigilancia de um especifico evento adverso à saúde é composta ao menos por dois subsistemas: ${ }^{4}$

1.Subsistema de informações para a agilização das ações de controle: situa-se nos niveis locais dos serviços de saúde e tem por objetivo agilizar o processo de identificação e controle de eventos adversos à saúde. A equipe que faz parte desse subsistema deve estar perfeitamente articulada com a de planejamento $e$ avaliação dos programas, responsável, portanto, pela elaboração das normas utilizadas no nivel local dos serviços de saúde.

O termo norma deve ser entendido no sentido de um instrumento para planejamento $e$ avaliação de programas de savide e, portanto, adequado à realidade local, seja no que tange às características particulares do comportamento de especificos agravos em função da estrutura epidemiológica local, seja pelo perfil profissional e dimensões da equipe envolvida ou ainda, pelas instalações, equipamentos, tecnologias $e$ recursos orçamentários disponípeis para o desenvolvimento de ações de controle.

Por localizar-se no nivel local dos serviços de saúde, as atribuições desse subsistema, com grande freqüência será exercida pelo mesmo profissional ou grupo de profissionais responsáveis pela implementação das ações de controle. Tal fato não interfere na perfeita delimitação de ambos os instrumentos: a vigilância e as açôes de controle. Podemos exemplificar com a atuação do clinico que ao atender um paciente estabelece o diagnóstico com fundamento na clinica e o tratamento com fundamento da terapêutica ambas constituindo instrumentos distintos utilizados pela medicina.

2.Subsistema de inteligência epidemiológica: é especializado e tem por objetivo elaborar as bases técnicas dos programas de controle de especificos eventos adversos à saúde a partir da análise sistemática do comportamento desses eventos com fundamento no conbecimento científico e tecnólogico disponivel.

Ao falarmos em bases técnicas de um programa, estamos nos referindo à fundamentação de um programa que, diferente da norma, apresenta um caráter mais universal. Por exemplo, as bases técnicas para um programa de controle de difteria em Santa Catarina, na Babia, ou, talvez, num país longínquo da Ásia, são muito semelhantes; o que irá diferir é a norma, que deverá estar vinculada às características locais já citadas.

Outro objetivo do subsistema de inteligência epidemiológica é identificar lacunas no conbecimento científico e tecnológico, uma vez que, à medida que for acompanbando o comportamento de especificos eventos adversos à saúde na comunidade, poderá, eventualmente, 
detectar mudanças desse comportamento não explicadas pelo conbecimento cientifico disponivel. Identificada essa lacuna no conbecimento disponivel, é papel da inteligência epidemiológica induzir a pesquisa.

Esse subsistema exerce também a função de incorporar aos serviços de saude o novo conhecimento produzido pela pesquisa, com o objetivo de aprimorar continuamente as medidas de controle. Isso pode ser feito, incluindo esse novo conbecimento nas bases técnicas que são encaminhadas aos serviços de saúde na forma de recomendaçóes disseminadas por Boletins Epidemiológicos.

Esse subsistema constitui a ponte entre o subsistema de serviços de saúde e o subsistema de pesquisa do SNS à medida que pode assumir o papel de indutor de linhas de pesquisa que respondam a questões prioritárias ou de importância emergente em saúde pública e, por outro lado, incorpora o conbecimento produzido ao atualizar continuamente as bases técnicas dos programas de controle de doenças.

Na figura 1 apresentamos um esquema das interações entre os subsistemas de vigilancia, serviços de saúde e de pesquisa no SNS.

\section{Abrangência da vigilância no Sistema Nacional de Saúde - SNS}

Os sistemas de vigilância para especificos eventos adversos à saude pode assumir diferentes papéis e caracteristicas num sistema de saúde. Entre eles, os mais relevantes são: ${ }^{4}$

1. Os sistemas de vigilância para especificos eventos adversos à saude constituem o elo de ligação entre o subsistema de serviços de saúde e o de pesquisa do SNS.

2. Os sistemas de vigilância abrangerão quaisquer eventos adversos à saúde, poderão ser desenvolvidos nas formas ativa ou passiva e utilizarão todas as fontes de informações necessárias e disponiveis.

3. Podem ser entendidos também como a inteligência do SNS voltada ao estabelecimento das bases técnicas para as ações de controle de especificos eventos adversos à saúde.
4. O SNS deverá desenvolver tantos sistemas de vigilancia para especificos eventos adversos à saúde, quantos sejam os problemas prioritários de saude para os quais haja possibilidade de desenvolver programas nacionais, estaduais, regionais ou locais de controle. Por sua vez, os sistemas locais de saúde poderão ou não aderir a cada um desses sistemas, conforme suas prioridades e recursos disponiveis para desenvolver os programas de controle dos agravos correspondentes. Constituem excecoes as doenças de notificacão compulsória.

\section{Limitações de sistemas de vigilância}

Embora os sistemas de vigilância não precisem ser perfeitos para serem uteis, muitas vezes certas limitações impedem que esse instrumento tenha a utilidade necessária que justifique sua implementação. Os fatores que mais freqüentemente levam a limitações do desempenho de sistemas de vigilancia são: ${ }^{18}$

A-Subnotificação.

$B$ - Baixa representatividade.

$C$ - Baixo grau de oportunidade.

D- Inconsistência da definição de caso.

A subnotificação geralmente decorre de que, na maioria, os sistemas de vigilancia são passivos, situação encontrada mesmo em paises desenvolvidos. O elevado custo dos sistemas ativos torna sua aplicação indicada para situações especiais já citadas. É freqüente em sistemas passivos a subnotificação atingir niveis superiores a $50 \%$ impedindo muitas vezes a identificação de tendências, grupos ou fatores de risco e levando ao retardo ou mesmo a ausência de ações de controle. A subnotificação está freqüentemente relacionada $a::^{18}$

a) a falta de conbecimento, por parte dos profissionais de saúde, a respeito da importância $e$ dos procedimentos necessários para a notificação.

b) o desconbecimento da lista de doenças contempladas por sistemas de vigilância.

c) a ausência de adesão à notificação, pelo tempo consumido no preenchimento da ficha e pela ausência do retorno da informação analisada com as recomendações técnicas pertinentes. 
Figura 1 - Esquema de interações entre os subsistemas de vigilância, serviços de saúde e pesquisa

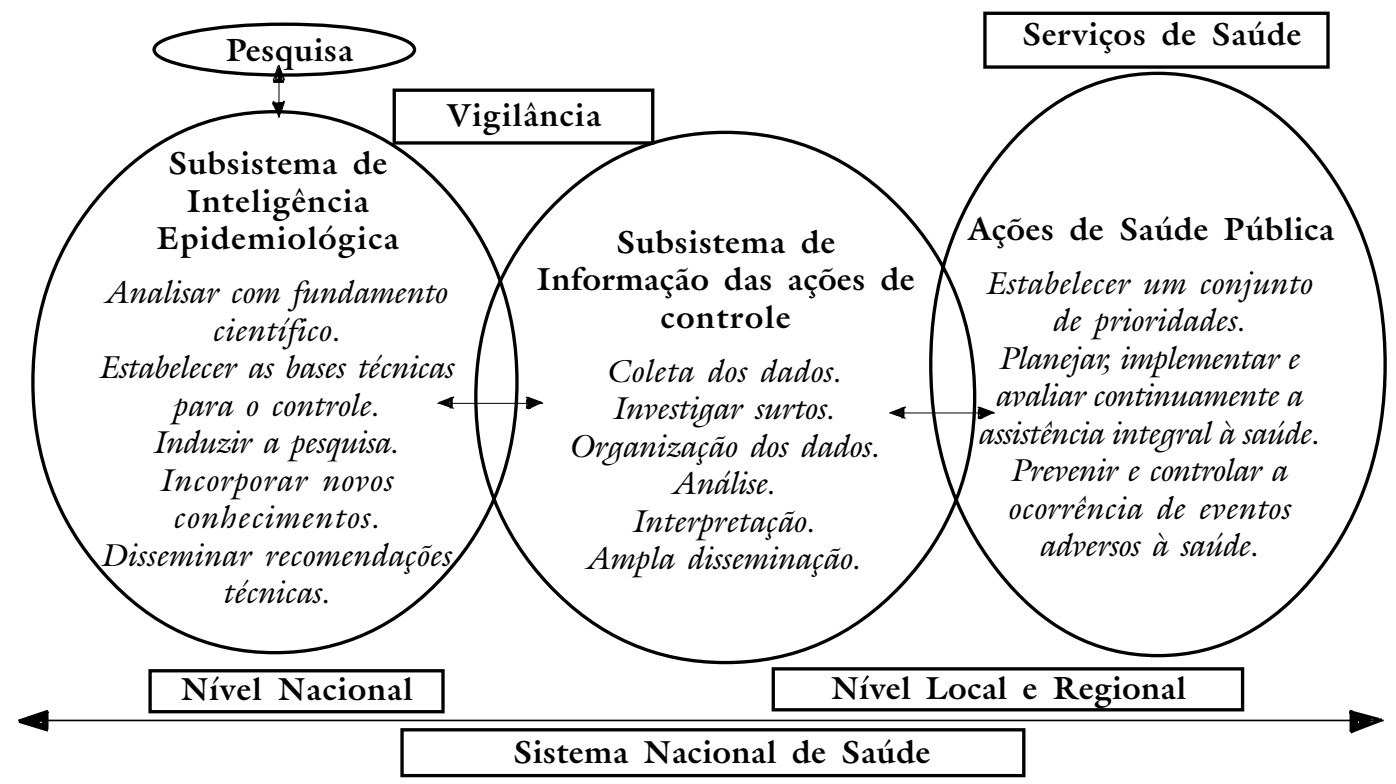

d) a preocupação dos profissionais de saúde com referência à quebra da confidencialidade das informações.

e) a falta de percepção, pelos profissionais, a respeito da releváncia em saúde pública das doenças submetidas à vigilância.

A baixa representatividade pode resultar da falta de homogeneidade da subnotificação na população alvo do sistema de vigilância, dificultando a identificação de tendências, grupos e fatores de risco. Os dois erros sistemáticos mais observados são: ${ }^{18}$

a) tendência a notificar mais os casos de maior gravidade e os hospitalizados do que os de caracteristicas benignas, ainda que estes uiltimos possam constituir as principais fontes de infecção;

b) notificação com maior intensidade de doenças que estão sendo focalizadas pelos meios de comunicação.

O baixo grau de oportunidade de um sistema de vigilância pode ocorrer em diferentes momentos por diversos motivos, entre eles: ${ }^{18}$ a) dificuldade, em alguns casos, de se obter o diagnóstico antes da confirmação laboratorial.

b) ineficiência dos serviços no procedimento de notificação.

c) demora na análise, problema freqüente quando o sistema de vigilancia é mais uma rotina burocrática do que uma atividade técnica voltada ao apoio dos serviços de saúde.

d) retardo que, em qualquer fase do sistema leva a uma demora na disseminação da informação analisada, impedindo que a população e os profissionais de saúde tenham as informações indispensáveis para uma ação oportuna e eficiente.

A inconsistência da definição de caso leva a vigiláncia a confirmar os casos aceitando o diagnóstico dos clinicos, independentemente da forma como eles foram efetuados.

Medidas visando ao aprimoramento de sistemas de vigilância

A participação dos médicos e demais profissionais de saúde é um ponto critico na qualidade da coleta de dados; portanto, o 
esclarecimento dessas equipes, salientando a importância da notificação de doenças para o aprimoramento dos serviços deve ser prioritário na formação e treinamento de recursos humanos. Porém, a adesão dos médicos e dos profissionais de saúde à notificação sistemática está, em boa parte, condicionada à resposta da vigilância, ou seja, à freqüência e agilidade com que ela devolve a esses profissionais as informações devidamente analisadas, acrescidas de recomendações técnicas úteis ao aprimoramento dos serviços de assistência à sauide.

A ampla utilização da vigilância pelo SNS pressupõe a existência de programas continuados de formação de epidemiologistas com treinamento especifico em vigilància. $O$ treinamento intensivo em investigação de surtos é obrigatório para a plena habilitação desse profissional. Talvez seja possivel afirmar que o principal vínculo da vigilancia ao método epidemiológico se estabeleça, justamente, nos procedimentos desenvolvidos numa investigação de surto.

$O$ aprimoramento das técnicas de investigação de surtos, nestes últimos 40 anos, se fez pela sua aplicação nas atividades da vigiláncia. A ênfase conferida às técnicas de investigação de surtos, incluindo o indispensável apoio laboratorial, já constituia a principal caracteristica dos programas pioneiros de adestramento de epidemiologistas para atuarem na vigilância, desenvolvidos na década de $50 .{ }^{19}$

Uma sólida infra-estrutura de laboratório é obrigatória para a eficiência da vigilância. Laboratórios capacitados pela incorporação continua de novas tecnologias de diagnósticos e para a pesquisa rotineira de marcadores biológicos de microrganismos e parasitas associados a formas particulares de comportamento de doenças na comunidade, constituem instrumento indispensáveis ao bom desempenho da vigilância.

O uso da monitorização em saúde pública

\section{Conceitos}

O termo monitorização, introduzido recentemente no idioma português, significando "acompanhar e avaliar" ou "controlar mediante acompanbamento", é usado em textos técnicos na área de saude com o mesmo significado da palavra em idioma inglês "monitoring", ou seja, "controlar e às vezes ajustar programas" ou "olhar atentamente, observar ou controlar com propósito especial” (Webster's Dictionary).

De acordo com Last, $^{20}$ o termo monitorização pode ser entendido no campo da saúde pública como:

a) "Elaboração e análise de mensurações rotineiras visando detectar mudanças no ambiente ou no estado de saúde da comunidade. Não devendo ser confundida com vigilancia. Para alguns monitorização implica intervenção à luz das mensuraçôes observadas";

b) "Contínua mensuração do desempenho do serviço de saúde ou de profissionais de saúde, ou do grau com que os pacientes concordam com ou aderem às suas recomendaçôes";

c) "Em administração, a contínua supervisão da implementação de uma atividade com o objetivo de assegurar que a liberação dos recursos, os esquemas de trabalho, os objetivos a serem atingidos $e$ as outras ações necessárias estejam sendo processadas de acordo com o planejado".

Lucas, ${ }^{21}$ de certa forma, apresenta uma conceituação de monitorização quando refere que a vigiláncia é também usada no planejamento da estratégia para o controle de doenças, enquanto a monitorização é aplicada no desenvolvimento de programas. Neste ponto, esse autor de certa forma concorda com o conceito de Last ${ }^{20}$ ao conferir um vínculo maior entre a monitorização e as ações de controle.

A Organização Mundial de Saúde, em publicação relativa a Programas Nacionais de Prevenção e Controle da AIDS, conceitua monitorização como o "contínuo acompanbamento das atividades de forma a garantir que as mesmas sejam desenvolvidas de acordo com o planejado". ${ }^{22}$ 
Ao analisar os diferentes conceitos de monitorização apresentados, verificamos que como instrumento aplicado à saúde pública ele ainda está em fase de desenvolvimento, mas a importância de sua aplicação nesta área é facilmente perceptivel. Ficam delineadas também algumas diferenças entre vigilancia e monitorização à medida em que esta última não apresenta um vinculo exclusivo com a epidemiologia e com a própria saúde pública, ainda que seja visivel sua aplicação nessas duas áreas.

\section{Semelhanças e diferenças entre vigilância e monitorização}

Talvez possamos identificar as principais diferenças entre vigilância e monitorização nos seguintes pontos:

a) A vigilância, por definição, acompanba o comportamento de especificos eventos adversos à saúde na comunidade, enquanto a monitorização trabalha especificamente com indicadores, sejam eles de sauide, demográficos, econômicos, sociais, de qualidade ambiental ou de serviços, etc.;

b) A vigilância é um instrumento exclusivo da saúde pública, enquanto a monitorização pode ser aplicada em diferentes campos de atividade;

c) A vigiláncia se caracteriza como uma aplicação do método epidemiológico enquanto, na monitorização, esse vínculo não é obrigatório;

d) A vigilância, por preocupar-se exclusivamente com o controle de doenças, é um instrumento de aplicação mais restrita em saúde pública do que a monitorização, que pode ser aplicada, por exemplo, na análise de situação de savide, constituindo esta última, talvez, o principal instrumento para a identificação de prioridades para politicas no setor saude.

e) O perfil dos recursos humanos para o uso de ambos os instrumento é também distinto. A vigilancia, por focalizar especificamente o controle de eventos adversos à saúde necessita de profissionais da área biomédica. Nas atividades de campo, especialmente em investigações de surtos o profissional médico $e$ de enfermagem constituem elementos indispensáveis da equipe. Conbecimentos da clinica, da epidemiologia e de aspectos relativos a fisiopatogenia, imunologia e dos fundamentos técnicos dos procedimentos diagnósticos da doença objeto da vigilância são pré-requisitos para a investigação de surtos e adequada interpretação das informações obtidas pela vigiláncia. Na monitorização o perfil profissional da equipe vai depender dos indicadores que estão sendo acompanhados. Se, por exemplo, estivermos monitorizando contaminação ambiental por substáncias ionizantes talvez o perfil mais adequado seja o de um físico; se estivermos monitorizando contaminação de estuários por metais pesados, a equipe ideal deverá contar com químicos e biólogos, ou seja, a monitorização acompanha o espectro multiprofissional da própria saúde pública.

f) As caracteristicas das instalações $e$ equipamentos necessários também diferem grandemente. Se, por exemplo estivermos monitorizando indicadores sociais, demográficos e de saude, possivelmente um bom suporte de informática que permita acessar e processar grandes bancos de dados seja suficiente. No entanto, se o objetivo for monitorizar indicadores de qualidade de tecnologias médicas como, por exemplo, vacinas e medicamentos, será indispensável um forte e sofisticado apoio laboratorial.

g) A vigilância possui critérios explícitos e já suficientemente validados de avaliação de desempenho, o mesmo não acontecendo com a monitorização.

Possivelmente a principal semelhança entre vigilancia e monitorização seja o fato de constituir atividade contínua, composta obrigatoriamente por três componentes: a) coleta de dados; b) análise regular dos dados; c) ampla e periódica disseminação dos dados a todos que deles necessitam.

\section{Aplicações da monitorização em saúde pública}

Se quisermos tornar mais abrangente $o$ conceito de monitorização apresentado por 
Last, ${ }^{20}$ poderiamos propor as seguintes aplicações num SNS: ${ }^{4}$

a) Analisar, continuamente, indicadores relativos à eficiência e efetividade dos serviços de saude $e$ ao desempenho dos profissionais que neles trabalham, assim como indicadores epidemiológicos que reflitam o impacto desses serviços na saúde da população.

Os resultados obtidos devem ser amplamente divulgados pois servirão tanto para o controle interno dos serviços de saúde como de instrumento de controle social do mesmo.

b) Analisar, continuamente, indicadores de qualidade de produtos de consumo humano, de tecnologias médicas, do exercicio profissional na área biomédica e, ainda, de riscos ambientais, para oferecer subsidios às medidas pertinentes relativas à fiscalização $e$ educação sanitária e, também, identificar necessidades de pesquisas cientificas ou de desenvolvimento tecnológico para a solução de determinados problemas identificados, assim como recomendar alterações na legislação especifica.

Neste caso a monitorização deve ser aplicada com vistas à avaliação contínua de exposição da população a riscos determinados por consumo dos mais diferentes produtos, pelo uso de tecnologias médicas e por condiçóes ambientais, no sentido amplo do termo. $\underline{E}$ de todo aconselbável que a(s) agência(s) responsável(eis) por esta atividade não tenha $(m)$ atribuicões no campo da fiscalizacão sanitária, para garantir sua independência.

c) Analisar, continuamente, indicadores de morbidade e mortalidade que permitam detectar alterações que expressem modificações nas condições de saúde da comunidade, buscando identificar suas causas e caracterizar perfeitamente seus efeitos. Identificadas as causas e caracterizados os efeitos pode, se indicado, sugerir o desenvolvimento de sistemas de vigilância para esse(s) especifico(s) evento(s) adverso(s) à saúde.

Nesse caso, a monitorização tem também por objetivo estabelecer a magnitude e o grau de prioridade de eventos adversos à saúde. d) Analisar continuamente indicadores demográficos, econômicos sociais e de saúde com vistas a subsidiar o estabelecimento de politicas no setor social e, em especial no da saúde.

e) Coletar e analisar, sistematicamente, informações pertinentes a programas de controle de especificos eventos adversos à saúde $e$ de seu impacto na incidência e prevalência do agravo objeto do programa, visando indicar, com base nas recomendaçôes técnicas disponiveis ou normas já elaboradas, as medidas imediatas de controle. Nesse caso, a monitorização é um instrumento da vigilância quando aplicada no subsistema de informação para agilização das açôes de controle.

f) Manter o contínuo acompanbamento das atividades meio previstas no ámbito dos serviços de saude, para assegurar os recursos humanos, materiais e financeiros necessários, a fim de que as atividades, programas ou projetos previstos sejam desenvolvidos conforme o planejado.

Características gerais da aplicação da monitorização em saúde pública

As atividades de monitorização desenvolvidas no ámbito dos serviços de saúde deverão ter as seguintes características:

1. A monitorização é um instrumento de aplicação obrigatória do subsistema de serviços de saude em todos os seus niveis.

2. As atividades de monitorização não apresentam, obrigatoriamente, complexidade técnica crescente do nivel local ao central dos serviços de sauide. Elas diferem pela abrangência com que se efetua a agregação dos dados e, muitas vezes, pelos indicadores utilizados.

3. Os profissionais ou equipes responsáveis por essa atividade em sistemas locais de sande serão os mesmos, ou trabalharão de forma integrada com aqueles que tem por atribuição a vigilancia, o planejamento e avaliação dos serviços de saúde. Já para as equipes que aplicam este instrumento nas Secretarias Estaduais e no Ministério da Saude seria recomendável sua integração a agências que centralizem a 
aplicação da epidemiologia (Centros de Epidemiologia).

4. As atividades de monitorização podem ser caracterizadas como a inteligência do SNS, oferecendo não só as bases para a sua avaliação técnica e operacional, mas também servindo de instrumento para a identificação precoce de alterações das condições de saúde da população $e$ de alterações ambientais que constituam riscos à comunidade e, ainda, para o contínuo controle sanitário da qualidade de produtos de consumo bumano, de tecnologias médicas e de serviços prestados à comunidade, na área da sauide.

A aplicação da monitorização na prática epidemiológica dos serviços de saúde

Desde meados da década de 80, tem sido amplamente aceita a existência de ao menos quatro grandes áreas de aplicação da epidemiologia nos serviços de saúde: ${ }^{23}$

1. Vigilância em saúde pública (ou epidemiológica, qualificativo ainda utilizado $\mathrm{em}$ nosso pais);

2. Análise da situação de saúde;

3. Identificação de perfis e fatores de risco;

4. Avaliação epidemiológica de serviços.

A vigilância como aplicação da epidemiologia em serviços de saúde já foi apresentada neste texto, motivo pelo qual passaremos diretamente a aplicação da monitorização na análise da situação de saúde, na avaliação epidemiológica de serviços e, por fim, na identificação de perfis e fatores de risco.

\section{A monitorização e a análise da situação de saúde}

$A$ análise epidemiológica de indicadores demográficos e de morbi-mortalidade com o objetivo da elaboração dos chamados "diagnósticos de saude", é uma prática antiga em nosso meio. Por vários motivos, nas últimas décadas, essa atividade foi sendo expressivamente reduzida no pais, com evidentes prejuizos ao adequado desempenho dos serviços de saúde. Felizmente, porém, nos últimos anos temos assistido a várias iniciativas preocupadas em retomá-la. Um exemplo recente é a iniciativa da Organização Pan-Americana de Saúde (OPAS) e do Ministério da Saúde por meio da proposta de criação da "Rede de Informações para a Saúde” (RIPSA), incentivando a utilização mais ampla da epidemiologia por meio do acompanbamento e análise sistemática da evolução de indicadores demográficos, sociais, econômicos e de saúde, com vistas a melhor compreensão dos determinantes das condiçôes de saúde da população.

As perspectivas de sucesso dessas iniciativas têm sido consideravelmente ampliadas pelo esforço dos organismos governamentais responsáveis por levantamentos e gerência de grandes bases de dados, para aprimorá-los, assim como facilitar o acesso aos potenciais usuários.

$O$ fortalecimento desta prática epidemiológica no SNS é indispensável para a melhor fundamentação das politicas no setor saúde. A simples inspeção da evolução de alguns indicadores apresentados na Tabela 1 nos oferece uma idéia das profundas modificações verificadas em nosso país, nos uiltimos 15 anos, $e$ das repercussões que essas mudanças deveriam determinar nas prioridades a serem seguidas.

Esse quadro de continuas e significativas modificações desses indicadores salienta a relevancia de capacitarmos os recursos humanos que atuam ou que virão a atuar nos serviços de saúde, na análise e interpretação de sua evolução à luz, por exemplo, de conceitos como o de "transição epidemiológica", que busca compreender as profundas mudanças nos padrôes de morbi-mortalidade ocorridos nas últimas décadas.

A evolução desse cenário deve ser acompanbada com atenção por todos os profissionais que assessoram ou decidem a respeito de politicas de saúde. Citariamos, como exemplo, o processo de envelhecimento da população e suas implicações nas caracteristicas da demanda dos serviços de saude, que geram necessidades de desenvolvimento de novas especialidades e de modificações na infra- 
estrutura e equipamentos dos serviços de saúde. Recentemente, Monteiro e colaboradores ${ }^{24}$ elaboraram interessante análise a respeito da melhoria dos indicadores de saude associados à pobreza no Brasil nos anos 90. Nesse trabalho, os autores mostram que indicadores intimamente relacionados à pobreza - como a mortalidade infantil $e$ a desnutrição nos primeiros anos de vida - evoluiram de forma continuamente favorável, nesse periodo, em todo o pais. No entanto, salientam que os indicadores de saude observados no Nordeste urbano estão ainda distantes daqueles observados nas cidades do Centro-Sul. Além disso observam que os progressos registrados no Nordeste Rural nas duas últimas décadas são menores do que os registrados no CentroSul Rural acarretando uma ampliação das desigualdades destas regiões.

A introdução nas rotinas dos serviços de saúde da monitorização de indicadores demográficos, sociais, económicos e de savide, acrescidos da análise e disseminação periódica desses dados analisados à luz do método epidemiológico, permitirá o aprimoramento da aplicação dos recursos disponiveis e um maior impacto dos programas desenvolvidos.

A monitorização e a identificação de perfis e fatores de risco

A urbanização e a industrialização determinaram um aumento da importáncia de uma série de riscos ambientais (contaminação da água e do ambiente por pesticidas e metais pesados, poluição do ar, riscos ocupacionais, etc.) e de condicionantes sociais e culturais que podem contribuir positiva ou negativamente nas condições de saúde das populações. Por outro lado, o desenvolvimento de novas e sofisticadas tecnologias médicas tem elevado sobremaneira o custo dos serviços, tornando indispensável a utilização racional dos recursos.

A epidemiologia pode ajudar a responder a esses novos desafios por meio da avaliação dos fatores condicionantes do processo saúde/doença, por meio da identificação de fatores de risco e de grupos da população mais vulneráveis (grupos de risco) a determinados agravos à saúde.

Essa contribuição da epidemiologia torna possivel o desenvolvimento de programas de saude mais eficientes, permitindo maior impacto das ações voltadas à assistência integral à saúde.

A monitorizacão de indicadores de qualidade de produtos de consumo bumano, de tecnologias médicas, do exercicio profissional na área biomédica $e$, ainda, de riscos ambientais incluindo os profissionais constitui instrumento que pode contribuir para a identificação de perfis e fatores de risco.

Tabela 1 - Evolução de alguns indicadores sociais, demográficos e de saúde no Brasil, nas décadas de 1980 e 1990

\begin{tabular}{l|c|c}
\hline \multicolumn{1}{c|}{ INDICADORES } & $\mathbf{1 9 8 0}$ & DÉCADA DE 1990 \\
\hline Populaçao Urbana (\%) & $67,5 \%$ & $78,4 \%(1996)$ \\
Taxa de Fecundidade & 4,3 & $2,3(1996)$ \\
Crescimento População Anual (\%) & $2,5(1970 / 1980)$ & $1,4(1991 / 1996)$ \\
Pop. de < 5 anos (em milhóes) & 16,4 & 15,6 \\
População Analfabeta => 10 anos & $25,3 \%$ & $16,2 \%(1995)$ \\
\% de Domicílios com Água & $53,3 \%$ & $84,3 \%(1996)$ \\
Mortalidade Infantil Proporc. p/Diarréias (\%) & 24,5 & $9,7(1992)$ \\
Desnutrição em < 5 anos (\%) & $18,4(1975)$ & $5,9(1996)$ \\
Mortalidade Proporc. p/Doenças Infecciosas & 9,3 & $4,7(1992)$ \\
PIB "per capita" (em R\$) & $3510(1985-1989)$ & $3460(1992 / 1996)$ \\
\% Idosos (60 anos e +) na População & $6,1 \%(1985-1989)$ & $7,4 \%(1992 / 1996)$ \\
Razão de Dependência & 0,73 & 0,58 \\
Renda familiar "per capita" (em R\$) & $276(1985-1989)$ & $195(1992 / 1996)$ \\
\hline
\end{tabular}

Fontes: Fundação Instituto Brasileiro de Geografia e Estatistica (IBGE); PNDC-1996. 


\section{A monitorização e a avaliação epidemiológica de serviços}

A avaliação de serviços de saúde pode ser feita de diversas formas, mas, de maneira geral, leva em conta o acesso da população aos serviços e a cobertura oferecida (exemplo: proporção de crianças vacinadas; proporção de individuos atingidos por determinada doença que são tratados $e$ acompanhados, proporção de gestantes inscritas e acompanhadas pelo programa, etc.), ou seja a proporção da população coberta por diferentes programas. É evidente que a cobertura somente será elevada se o acesso for amplo. ${ }^{25}$

$A$ avaliação de um programa desenvolvido por um sistema local de saúde pode ser efetuada verificando quais das atividades previstas foram implementadas com êxito. Outra maneira de efetuá-la é verificando o impacto do plano na evolução de indicadores de saúde ou na freqüência dos agravos à saúde contemplados pelo plano.

Apresentando-se de forma simplificada o processo de avaliação de serviços, podemos apontar os seguintes passos: ${ }^{25}$

1. Selecionar indicadores mais apropriados, de acordo com os objetivos do programa.

2. Quantificar metas a serem atingidas com referência aos indicadores selecionados.

3. Coletar as informações epidemiológicas necessárias.

4. Comparar os resultados alcançados $\mathrm{em}$ relação às metas estabelecidas.

5. Revisar as estratégias reformulando o plano, quando necessário.

A contribuição da epidemiologia nesse processo se dá, principalmente, na seleção, construção e análise dos indicadores e na análise do impacto, em termos de morbi-mortalidade das doenças contempladas pelo plano.

Em sintese, pode-se dizer que esse processo visa estabelecer a efetividade e a eficiência dos serviços de saúde, entendendose por eficiência a capacidade de um programa alcançar os resultados pretendidos despendendo um minimo de recursos e efetividade como a habilidade de um programa produzir os resultados esperados nas condições de campo. Devemos lembrar que a efetividade é um atributo distinto de eficácia que é medida pela capacidade de um programa produzir resultados em condições ideais.

$\mathrm{Na}$ avaliação epidemiológica de serviços aplica-se a monitorizacão de indicadores velativos ì eficiencia e efetividade dos servicos de savide e ao desempenho dos profissionais que neles trabalham, assim como indicadores epidemiológicos que reflitam o impacto desses servicos na savide da populacão.

\section{Considerações finais}

$O$ uso da epidemiologia nas práticas sanitárias não é novo em nosso pais. O Estado de São Paulo, por exemplo, em 1894, iniciava $o$ acompanhamento de estatisticas vitais e, a partir dos anos 20, já contava com um sistema de informação referente a doenças de notificação compulsória razoavelmente bem estruturado. ${ }^{26}$

No entanto, foi com a incorporação da vigilância às atividades regulares dos serviços de saúde pública, na segunda metade da década de 70, que obtivemos avanços mais significativos na criação e fortalecimento no pais, dos instrumentos indispensáveis para uma utilização mais ampla da epidemiologia. Apesar das dificuldades no processo de implantação da vigilância, são inegáveis os avanços alcançados, merecendo destaque a(o): ${ }^{4}$

a) definição de critérios nacionais para identificação de prioridades no estabelecimento das doenças de notificação compulsória e na padronização de fichas de notificação;

b) aprimoramento da qualidade da informação de morbidade relativa às doenças transmissiveis de notificação compulsória;

c) implantação do Sistema Nacional de Informação de Mortalidade com a padronização do formulário de declaração de óbito para todo $o$ pais;

d) elaboração de indicadores de mortalidade $e$ morbidade para o continuo acompanhamento, com o objetivo de identificar mudanças no comportamento das doenças, inclusive o aparecimento de agravos inusitados; e também 
de indicadores operacionais visando avaliar o desempenho desse sistema de informação; $e$

e) implantação do Sistema Nacional de Laboratórios de Saúde Pública com a criação da figura do Laboratório Nacional de Referência para doenças especificas ou para grupo delas.

No entanto, a despeito dos reflexos positivos da incorporação e consolidação da vigilância como uma das principais práticas sanitárias em nosso país, ela enfrenta, até hoje, dificuldades para manter a sua regularidade e qualidade, em virtude, principalmente, dos seguintes fatores:

a) falta de uma rede básica de serviços de sauide bem estruturada;

b) politicas institucionais de longo prazo, mal definidas, criando obstáculos ao estabelecimento de diretrizes sólidas para à formação e reciclagem de recursos humanos $e$ criação de carreiras estáveis; $e$

c) ausência de um programa regular, de conteuido mais denso tanto na parte teórica como na de campo, voltado à formação de epidemiologistas capacitados a atuar nas principais áreas de aplicação da epidemiologia em serviços de sauide.

Um aspecto que surpreende ao analisarmos o processo de incorporação da vigilância em nosso sistema de saúde diz respeito ao fato de que associado, direta ou indiretamente, à sua implantação, tivemos a significativa fragilização das demais aplicações da epidemiologia nos serviços de saúde, mesmo em Estados aonde a utilização da epidemiologia datava do inicio do século.

A busca da explicação desse efeito, a primeira vista paradoxal, constitui tarefa árdua pois lidará com questões complexas. Porém, se analisarmos as considerações feitas por Langmuir $^{8}$ nas suas primeiras tentativas de conceituar a vigilancia, citadas no inicio deste texto, talvez possamos obter algumas pistas interessantes na busca de respostas a essa questão.
Analisando a forma pela qual a vigilância foi incorporada em nosso pais e possivelmente nos demais paises da América Latina, verificaremos que não nos preocupamos em identificar as fronteiras entre vigilancia e ações de controle. Possivelmente isso se deva, ao menos em parte, à grande influência de nossa experiência na utilização desse instrumento durante a Campanba de Erradicação da Variola.

Para a organização da Campanba, cada pais correspondia ao que seria hoje o nivel local de um SNS e o conceito de vigilancia aplicado nas Unidades Nacionais era de um sistema de informação para a agilização das açôes de controle. Isto justificava-se, uma vez que a vigilância entendida como inteligência epidemiológica segundo o conceito de Langmuir - era desenvolvida em Genebra, junto ao comando da Campanha.

A falta de uma nitida delimitação entre vigilância e ações de controle e a organização centralizadora dos serviços de saúde, levaram as equipes responsáveis pela vigilância em nosso pais a assumirem a atribuição de coordenação dos programas de controle das doenças transmissiveis. Tal responsabilidade, por suas caracteristicas, absorveram totalmente sua capacidade de trabalho, uma vez que deveriam responder às necessidades sentidas pela população de controlar doenças que freqüentemente se apresentam na comunidade na forma de epidemias; necessidades que sempre excedem os recursos disponiveis.

Absorvidas pelas responsabilidades de coordenação de programas, as equipes que atuavam na vigilància raramente conseguiram estruturá-la abrangendo os três componentes obrigatórios desse instrumento: a informacão; a análise e a ampla disseminacão da informaça analisada a todos que dela necessitam. Com isso, a vigilância caracterizou-se mais como um sistema de informação para apoiar a coordenação de programas de controle de doenças infecciosas do que um instrumento de apoio técnico dos serviços de saúde, fundamentado em conbecimento cientifico $e$ tecnológico rigorosamente atualizado ou, em 
outras palavras, não assumiu o papel de ponte entre a produção do conbecimento científico $e$ tecnológico e os serviços de saude pública.

Por outro lado, não conseguimos também estabelecer os limites nitidos entre a vigiláncia $e$ as demais aplicaçôes da epidemiologia nos serviços de saúde. Essa falta de clareza levou com alguma freqüência ao esvaziamento dos serviços de epidemiologia, pela progressiva transferência dos epidemiologistas para as equipes de vigilancia. Como resultado, observamos a fragilização de antigos serviços de epidemiologia que, com maior ou menor eficiência, funcionavam desde o final do século passado em algumas Unidades da Federação, além de não criarmos, até recentemente, condições para seu desenvolvimento aonde eles não existissem.

Tais fatos reforçam as justificativas da adoção da denominação, já consagrada internacionalmente, de vigilância em saúde pública ou simplesmente vigilância, $\mathrm{em}$ substituição à vigilância epidemiológica. Nos parece claro o fato do qualificativo epidemiológica ter induzido a equivocos que, de certa forma, contribuiram para o enfraquecimento da aplicação mais ampla da epidemiologia em serviços no Brasil. Reforça o argumento favorável a adoção de terminologia internacionalmente aceita o fato dessa padronização em áreas técnicas facilitar tanto a incorporação como a disseminação de novos conbecimentos.

Há, porém, uma outra questão envolvida na fragilização da epidemiologia nos serviços de saúde; ela está ligada à excessiva ênfase conferida à assistência médica na fase inicial da implantação do SUS, em detrimento dos programas de saúde que têm por característica fundamentar-se em critérios epidemiológicos para o estabelecimento de prioridades.

De certa forma, a necessidade do uso mais amplo da epidemiologia em nossos serviços de saúde foi percebida pelos sanitaristas já em meados dos anos 80, quando a atuação da vigilancia era criticada por preocupar-se exclusivamente com doenças e não com as condições de saúde e bem estar da população. Tais criticas, de alguma forma, constituiram as origens de algumas propostas que, freqüentemente, receberam a denominação de vigilancia à sauide, implementadas em algumas administraçôes municipais progressistas a partir da redemocratização do país.

$\mathrm{Na}$ realidade, o que estávamos verbalizando, não explicitamente, eva justamente a necessidade da ampla utilização da epidemiologia em nossos serviços de saúde, para que, nela fundamentada, pudéssemos implementar programas integrais e polivalentes de sauide, aceitando, portanto, com adaptações, as propostas da Conferência de Alma Ata. ${ }^{27}$

Outro aspecto importante a ser assinalado é o de que a vigiláncia em nosso país, não acompanhou o desenvolvimento técnico deste instrumento, mantendo até hoje, em suas linhas gerais, a proposta implementada pelo Ministério da Saude a partir de 1975. Isso dificultou, durante as discussóes voltadas à implantação do SUS, o desenvolvimento de propostas inovadoras e mais abrangentes para sua aplicação.

Neste momento em que assistimos o fortalecimento do processo de municipalização dos serviços e a rediscussão dos papéis dos niveis estaduais e federal no ámbito do SUS e, por outro, ao aparecimento de condições objetivas para a ampliação e modernização da vigilancia $e$ das demais práticas da epidemiologia em nosso pais, é importante a delimitação das áreas de aplicação da epidemiologia em todos os niveis do sistema de saúde e a perfeita identificação dos diferentes perfis de profissionais que deverão atuar em cada uma delas, assim como da infra-estrutura, instalações e equipamentos necessários.

Essa preocupação é fundamental para a definição de politicas institucionais de longo prazo que, por sua vez, constituem pressuposto para o desenvolvimento e a implementação de programas de formação e capacitação de epidemiologistas que deve privilegiar não só o treinamento de profissionais que já ingressaram no serviços de saúde mas, também, estabelecer 
uma estratégia de formação de uma nova geração de epidemiologistas de campo que constitua massa critica de dimensão suficiente para alcançarmos, a médio prazo, um salto de qualidade neste campo.

Concluindo, cumpre assinalar que a estratégia para atingir um grau apreciável de auto-sustentação do SUS está em boa parte condicionada à nossa capacidade de fortalecer a pesquisa em saúde pública, à semelhança do que fizeram paises cujos sistemas de saúde alcançaram bom desempenho. Analisando o desempenho da saúde pública brasileira dos primórdios deste século verificaremos que a pesquisa foi um dos três pilares que deram sustentação ${ }^{26}$ à nossa bem sucedida experiência e esse resultado foi atingido graças à nossa capacidade de criar um processo bem articulado de indução, produção e consumo do conbecimento produzido. ${ }^{28}$

Hoje, tanto a sociedade brasileira como nosso sistema de saúde, são muito mais complexos do que na era de Oswaldo Cruz e Emilio Ribas, mas guardadas as devidas proporções, a análise desse periodo pode nos oferecer subsidios para atingirmos um desempenho semelhante. Com esse objetivo, o uso da vigilância e da monitorização, com a abrangência apresentada neste texto, constitui peça fundamental, pois são instrumentos eficientes tanto na identificação de lacunas no conbecimento a respeito do comportamento de especificos agravos como de prioridades em saude pública, além de cumprirem o papel de indutores da pesquisa e incorporadores do conbecimento produzido. Portanto, sua ampla utilização permitiria politicas sociais no setor saúde mais adequadas à nossa realidade $e$ necessidades, fortaleceria a capacidade do SUS de responder a situações inusitadas e de relevância emergente, assim como de aprimorar continuamente os serviços de saúde incorporando novos conbecimentos científicos e tecnológicos.

\section{Bibliografia}

1. Fossaert DH, Llopis A, Tigre CH. Sistemas de vigilancia epidemiológica. Boletin de 1a Oficina Sanitaria Panamericana 76:512-525, 1974.

2. Doll R. Monitoring the national health service. Proceeding of The Royal Society of Medicine 66:729-740, 1973.

3. Editorial. Vigilância epidemiológica. International Journal of Epidemology, 5:4-6, 1976.

4. Waldman EA. Vigilância como prática de saúde pública. Tese de Doutorado. Universidade de São Paulo, São Paulo, 1991.

5. Romero A, Troncoso M. La vigilancia epidemiológica: significado e implicaciones en la práctica y en la docencia. Cuadernos de Medicina Social 17:17-28, 1981.

6. Langmuir AD. Willian Farr: founder of modern concepts of surveillance. International Journal of Epidemiology 5:13-18, 1976.

7. Schmid AW. Glossário de epidemiologia. Arquivo da Faculdade de Higiene de SãoPaulo $\mathbf{1}^{\mathbf{0}}$.(supl.):1-20, 1956.

8. Langmuir AD. Evolution of the concept of surveillance in the United States. Proceeding of The Royal Society of Medicine 64:681-684, 1971.

9. Langmuir AD. The surveillance of communicable diseases of national importance. New England Journal of Medicine 268:182-192, 1963.

10.Langmuir AD, Andrews JM. Biological warfare defence. 2 - The Epidemic Intelligence Service of the Communicable Disease Center. American Journal Public Health 42:235-238, 1952.

11. Nathanson N, Langmuir AD. The Cutter incident. Poliomyelitis following formaldehyde-inactivated poliovirus vaccination in the United States during the spring of 1955. I. Background. American Journal of Hygiene 78:16-28, 1963.

12.Raska $K$. The epidemiological surveillance programme. Journal of Hygiene and Epidemiology 8:137-168, 1964. 
13. Foege WH, Hogan RC, Newton LH. Surveillance projects for selected diseases. International Journal of Epidemiology 5:29-37, 1976.

14. Thacker SB, Berkelman RL. Public health surveillance in the United States. Epidemiology Review 10:164-190, 1988.

15. Teutsch S, Thacker SB. Planificación de un sistema de vigilancia en salud pública. Boletín Epidemiológico. Organización Panamericana de la Salud 16:17, 1995.

16. Waldman EA, Rosa TEC. Vigilância em saúde pública. Ed. F. Peiropolis, São Paulo, 1998 (no prelo).

17.Declich S, Carter AO. Surveillance health: historical origins, methods and evaluation. Bulletin of the World Health Organization 72:285-304, 1994.

18.Centers for Disease Control and Prevention. Principles of epidemiology. An introduction to applied epidemiology and biostatistics. $2^{\text {nd }}$ edition, 1992.

19.Langmuir AD. "The Epidemic Inteligence Service" of the Center for Diseases Control. Public Health Report 95:470-477, 1980.

20.Last JM. A dictionary of epidemiology. Oxford University Press, New York, 1988.

21.Lucas AD. Surveillance of communicable diseases in tropical Africa. International Journal of Epidemiology 5:39-43, 1976.

22. World Health Organization. Monitoring of national AIDS prevention and control programmes: guiding principles. WHO AIDS Series, $n$ 4. Geneva, p. 1-26., 1989.

23. Castellanos PL. Epidemiologia $y$ organización de los servicios. In: Organización Panamericana de la Salud. La formación en epidemiologia para el desarrollo de los servicios de salud. Washington, Publicación serie: Desarrollo de Recursos Humanos $N^{0} 88$, p. 30-40, 1987.

24. Monteiro CA, Benicio MHD, Freitas ICM. Melhoria em indicadores de saude associados à pobreza no Brasil dos anos 90: descrição, causas e impacto sobre desigualdades regionais. Núcleo de Pesquisas Epidemiológicas em Nutrição e Saúde da USP, São Paulo, 1997.

25. Vaughan JP, Morrow RH. Epidemiologia para os municipios. Manual para gerenciamento dos distritos sanitários. Editora HUCITEC, São Paulo,1992.

26. Mascarenhas RS. Contribuição para o estudo da administração sanitária estadual em São Paulo. Tese de Docência-livre. Universidade de São Paulo, São Paulo, 1949.

27. Glasunov IS, Grabauskas V, Holland WW, Epstein FH. An integrated programme for the prevention and control of noncommunicable diseases. Journal of Chronic Disease 36:419-426, 1983.

28. Stepan N. Gênese e evolução da ciência brasileira: Oswaldo Cruz e a politica de investigação científica e médica. Ed. Artenova, Rio de Janeiro, 1976. 